\title{
The Future - Part 1:
}

\section{Technological advances}

\section{$S$ Beningfield $M B C h B(U C T)$, FFRad(D)SA}

Professor and Head: Department of Radiology, University of Cape Town
B ased on the consistent and stellar evolution of our specialty over the last 30 years, it is virtually a certainty that there will be further dramatic breakthroughs in Diagnostic Radiology. The bets are off, however, when trying to predict what these changes could be. Certain general trends are fairly clear, including refinements of existing technology leading to smaller, faster and friendlier machinery with more facilities. Less striking but highly practical is the increasingly modular approach to machine design, permitting easy upgrading of equipment. More attention is also being paid to functional and quantitative evaluation. Invasive devices and procedures are more dependable, with their appropriateness in the clinical arena being clarified and formalised.

\section{Medical informatics}

Most information technology benefits to Radiology ride on the back of commercial and consumer demand. Ultra-high speed wireless connections will make it possible to report from any location, at any time. The transfer of all but the largest images will be possible in the magical time of two seconds, the time it takes to extract a film from its jacket and snap it up on the viewing box. The predicted demise of film, however, may take a lot longer than expected, and it may persist indefinitely in some hospitals.

Electronic patient records with organised, intelligent, integrated and confidential medical data storage have been identified as a key growth node, with good reason. This area is subject to intense interest at present, although the complexity is highly challenging. A watertight universal patient identifier system will be critical. Computer-aided diagnosis will probably take many years to achieve, and may then only serve as a support system, providing a cost-effective means of double-reading images. Images and reports (verbal or written) sent immediately to the referring clinician's desktop monitor will be the norm.

Cost-cutting pressures could be firmly implemented with instant computer analysis and preauthorisation. Closer integration of the various data standards is inevitable, with the combining of scheduling, clinical, imaging and financial information.

\section{Medical science}

Genetic and molecular advances appear set to dominate medicine in the next century. Will atherosclerosis and cancer vanish? Can viral illness be uniformly overcome? Patientcentric pressure will feature strongly in the directions taken by future research.

And what of the proposed ability to suspend aging by centromere intervention? Will families have to limit offspring to one person, in return for immortality? Will the usual diseases of the elderly manifest if aging is arrested at 30 ? 


\section{from page 6}

\section{The modalities}

Are we running out of new methods to view and guide our invasion of the interior of the human body? Although science appears to be approaching a more comprehensive understanding of atomic structure and physical phenomena, does this diminish the likelihood of spectacular advances? It is interesting to remember that the principles of CT and MRI were known for many years before they were applied to clinical imaging.

What about the other parts of the electromagnetic spectrum, such as the infrared, ultraviolet, and microwave wavelengths? And laser, electrical conductivity or forms of sound other than ultrasound? Could we see images based on subatomic particles or quantum phenomena, with the arrival of "Quark Tomography" or "Boson Resonance Imaging"?- surely no less strange than Positron Emission Tomography?

Direct digital acquisition, high acquisition speed, radiation dose reduction and real-time $3 \mathrm{D}$ post-processing are certain, as are many potential variations of functional and quantitative imaging.

\section{Viewing monitors and oper- ating consoles}

High-resolution, high-intensity, all-purpose workstations permitting reporting of all modalities are largely the consequence of commercial 3-dimensional data display and manipulation. Viewer location will be immaterial, and multi-reading by a number of radiologists may become the rule. These workstations could be head-mounted devices (possibly termed "headstations"), with low-power laser beams scanning highly detailed images directly onto the retina. The entire workstation could be a remotely connected wallet-sized unit.
Vendor-specific viewing and operating consoles could make way for software-based systems running on off-the-shelf hardware, facilitating generic replacements and upgrades.

\section{The "front end"}

The detector/acquisition system may become the single proprietary component of imaging equipment manufacturers. Vendors may focus exclusively on acquisition modules, leaving the data processing and display to standard computers run with customised software. The open standards paradigm led by the DICOM 3 protocols will hopefully lead to similar electromechanical standards, permitting compatibility for inter-vendor component and module interchange. For example, an integrated X-ray tube and generator in a shoebox-size unit could simply be clipped into position when replacement is required.

\section{X-rays}

Huge amounts of research and development finance have gone into attempting to design commercial direct digital systems. The problem in manufacturing large area detectors or alternate systems appears more challenging than initially thought. Some flat plate detectors exceed 15 kilograms, precluding their use as mobile receptors. For the time being, the intermediate technology photostimulable phosphor computed radiography (CR) systems dominate this sector.

\section{Angiography}

Although digital subtraction angiography appears to be holding its own against the onslaught of Magnetic Resonance Angiography and Computed Tomographic Angiography when intervention is planned, there appears to be little that is revolutionary on the horizon.

\section{Computed Tomography (CT)}

Virtual colonoscopy, bronchoscopy and other 3-dimensional displays could become the standard if consumer-driven demand escalates. Combined multipurpose fluoroscopic, angiographic and CT units should be possible when large flat panel detectors arrive. Cone Beam Computed Tomography (CBCT) using the full area of flat plate detectors for data acquisition in a CT gantry should allow almost instantaneous volumetric acquisitions.

\section{Ultrasound}

Three-dimensional ultrasound with volumetric display has yet to be widely used, but refinements of existing technology promise ongoing development. Modular replaceable ultrasound units integrated into interventive fluoroscopic and CT units should be standard issue.

\section{Nuclear Medicine}

Positron Emission Tomography (PET) and Single Photon Emission Computed Tomography (SPECT) imaging may merge further. Targeted imaging with monoclonal antibodies will be extended, paving the way for other organn-specific contrast agents.

\section{Magnetic Resonance Imaging}

Diffusion and perfusion imaging may be followed by the re-popularisation of metabolic imaging and spectroscopy. Ceramic MRI may resurface, but major developments could take a back seat to incremental improvements. Organ specific and targeted imaging 


\section{The Future - Part 1}

\section{from page 7}

are beginning to appear commercially, and may set the scene for the future.

\section{Interventive Radiology}

Smaller, smarter, slicker will be the bywords here, but political pressure and the relationship with other minimally invasive procedures may be the pivotal aspects. The number of invasive procedures may well expand, despite the increased involvement of clinical colleagues. This may be partly as a result of the incorporation of previously unguided procedures.

The promise of routine remotely-performed procedures appears a long way off, despite huge investments in the technology, particularly feedback transducers.

\section{The specialty}

Turf wars, clinical re-alignments, and reimbursement issues may overshadow technical advances. A major split of the specialty into the hands-on and remotely conducted components may occur, with centralised or home reporting facilitated for the latter.

Organ-specific teams will probably emerge from the turf wars, becoming the rule in the bigger centres. Medical training may adjust to this concept, with, for example, a career neuroradiologist or technique specialist commencing training straight after school, in the same way that dentistry splits from general medicine.
Could we witness a backlash against the sterile and remote digital environment, with close personal attention and professional interaction becoming a selling point for some centres, rather than technical prowess?

\section{Conclusion}

Tissue-specific imaging remains our unreachable objective. Non-harmful intervention is the ideal. In the attempt, many promising new technologies will go the way of kymography, electron radiography and thermography, while others, at present unknown, may dominate. Gradual stepwise progress will hopefully be interspersed by exciting new developments, sending us all back to being students again. 